\section{Brain lipid-binding protein is a direct target of Notch signaling in radial glial cells}

\author{
Todd E. Anthony, ${ }^{1}$ Heather A. Mason, ${ }^{2}$ \\ Thomas Gridley, ${ }^{3}$ Gord Fishell, ${ }^{2}$ and \\ Nathaniel Heintz ${ }^{1,4}$ \\ ${ }^{1}$ Laboratory of Molecular Biology, Howard Hughes Medical \\ Institute, The Rockefeller University, New York, New York \\ 10021, USA; ${ }^{2}$ Developmental Genetics Program and the \\ Department of Cell Biology, The Skirball Institute of \\ Biomolecular Medicine, New York University Medical \\ Center, New York, New York 10016, USA; ${ }^{3}$ The Jackson \\ Laboratory, Bar Harbor, Maine 04609, USA
}

Radial glia function during CNS development both as neural progenitors and as a scaffolding supporting neuronal migration. To elucidate pathways involved in these functions, we mapped in vivo the promoter for $B l b p$, a radial glial gene. We show here that a binding site for the Notch effector CBF1 is essential for all Blbp transcription in radial glia, and that BLBP expression is significantly reduced in the forebrains of mice lacking the Notch1 and Notch3 receptors. These results identify $B l b p$ as the first predominantly CNS-specific Notch target gene and suggest that it mediates some aspects of Notch signaling in radial glia.

Received February 2, 2005; revised version accepted March 25, 2005.

During the development of the mammalian brain, billions of neurons have to be generated, sent to their appropriate locations, and then interconnected in a precise manner. This task is particularly complex in the cerebral cortex, where an inside-out mode of development results in newly generated neurons having to migrate over larger distances through increasingly crowded terrain as neurogenesis proceeds (Angevine and Sidman 1961). Numerous studies have documented the critical role played by the radial glial scaffold in facilitating this migration, and estimates are that as much as $90 \%$ of neuronal migration in the cortex may take place upon radial glial cells (Sidman and Rakic 1973; Hatten 1999). In addition to their structural role, radial glial cells also function as neuronal progenitors (Malatesta et al. 2000; Miyata et al. 2001; Noctor et al. 2001; Tamamaki et al. 2001) and have been shown to be the source of most neurons throughout the CNS (Anthony et al. 2004).

The dual role played by radial glia as both migratory scaffolding and neuronal progenitors has suggested that there may be intimate links between the signaling pathways that control radial glial cell development, neuronal generation, and neuronal migration. The fact that mi-

[Keywords: Neuronal-glial interactions; cortex; cerebellum; Bergmann glia]

${ }^{4}$ Corresponding author.

E-MAIL heintz@rockefeller.edu; FAX (212) 327-7878.

Article and publication are at http://www.genesdev.org/cgi/doi/10.1101/ gad.1302105 grating neurons regulate morphological and proliferative characteristics of glia (Hatten 1985) has led to considerable focus on neuronally derived signals, and several cell surface and diffusible molecules have been implicated in neuronal-radial glial signaling, including Astrotactin (Zheng et al. 1996), Neuregulin/ErbB2 (Anton et al. 1997; Rio et al. 1997; Patten et al. 2003; Schmid et al. 2003), and Notch (Gaiano et al. 2000; Gaiano and Fishell 2002; Patten et al. 2003; Schmid et al. 2003). In contrast, the genes expressed within radial glia that mediate the radial glial response to neuronal signaling are not well characterized. One candidate is brain lipid-binding protein $(B l b p, F a b p 7)$, a gene that is dynamically regulated in radial glia by migrating neurons (Feng et al. 1994; Kurtz et al. 1994; Feng and Heintz 1995). BLBP is a member of the large family of hydrophobic ligand-binding proteins (FABPs), molecules that have been shown to modulate transcription through their interactions with nuclear receptors and to play roles in metabolism (Haunerland and Spener 2004). Immunoelectron microscopy has demonstrated that BLBP is present in both the cytoplasm and nucleus in vivo, suggesting that similar to other FABPs, BLBP may be involved in the trafficking of a ligand to a nuclear receptor (Feng et al. 1994). Consistent with its proposed role in mediating neuronal-glial signaling, antibody blocking experiments have shown that BLBP function is required for radial glial morphological changes in response to neuronal cues (Feng et al. 1994; Anton et al. 1997) and for regulating the morphology and axonal interactions of Schwann cells (Miller et al. 2003).

Previous analysis in transgenic mice has shown that migrating neurons induce Blbp transcription in glia through elements lying within the first $766 \mathrm{bp}$ of its promoter (Feng and Heintz 1995). In order to gain insight into the signaling pathways that induce Blbp, we have fine mapped the 766-bp Blbp promoter in transgenic mice. We report here that a single binding site in the 766-bp promoter for the Notch effector CBF1 is required for all Blbp transcription in the developing brain, and that BLBP expression is dramatically reduced in the forebrains of mice lacking the Notch1 and Notch3 receptors. These results identify Blbp as a direct target of Notch signaling in radial glia, and suggest that BLBP may mediate some aspects of glial responses to Notch.

\section{Results and Discussion}

Previous work has demonstrated that Blbp transcription is induced in radial glia by migrating neurons, and that the elements mediating this induction lie within the first 766 bp of Blbp 5' flanking genomic sequence (Feng and Heintz 1995). These and subsequent studies (Josephson et al. 1998) also showed that the Blbp promoter is modular, with distinct portions of the promoter being required for transcription in different regions of the CNS. In order to identify pathways involved in neuronal-radial glial interactions rather than region-specific factors, we chose to map deletion mutants at two different time points and locations; for this, we chose the embryonic day 12 (E12) forebrain and post-natal day 6 (P6) cerebellum since at these times BLBP is highly expressed in radial and Bergmann glia, respectively, and extensive radial glial-directed neuronal migration is taking place. Although the previous studies contained low magnifica- 
tion images of histochemical staining for transgene driven $\beta$-galactosidase, immunostaining to confirm the specificity of the 766-bp promoter was not included. To do this, we generated transgenic mice expressing enhanced green fluorescent protein (eGFP) under the regulation of the 766-bp promoter and immunostained founder mice at E12 and P6 for BLBP and eGFP. At both time points, eGFP expression was highly correlated with BLBP expression, and high magnification merged images demonstrated that expression was in the same cellular populations (Fig. 1). These results establish that the 766bp $B 1 b p$ promoter is sufficient to recapitulate endogenous BLBP expression in transgenic mice.

To map the inducible element(s) required for BLBP expression in radial glia, we assayed a series of promoter mutants with large deletions in transgenic mice (Fig. 2); from this initial analysis, several observations were made. First, sequences within the first 400 bp were sufficient to direct expression at both time points (construct 2), driving strong reporter activity in both E12 forebrain radial glia and P6 cerebellar Bergmann glia. Interestingly, although this construct drove full expression throughout the embryonic CNS, post-natal expression was reduced or absent in many brain regions. This indicates either that distinct combinations of pathways regulate embryonic and post-natal transcription of $B 1 b p$, or that transcription in some post-natal brain regions requires additional factors that are not essential embryonically.

Whereas promoters containing deletions between -400 and -500 (construct 3 ) as well as -100 to -230 (constructs 6 and 7) all retained strong activity, deletion of elements from -220 to -400 (constructs 4 and 5) ablated virtually all reporter expression both embryonically and post-natally. This established that elements between -230 and -400 are critical for Blbp transcription throughout the developing CNS. To determine what temporal

A
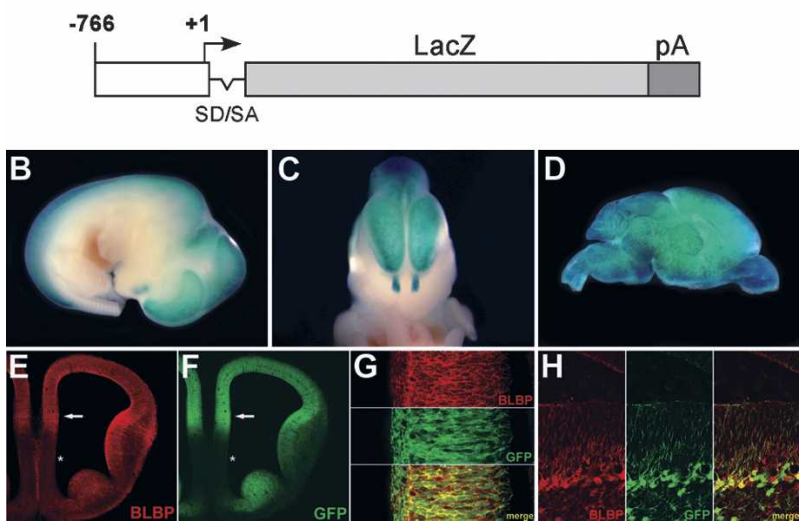

Figure 1. The 766-bp Blbp promoter recapitulates endogenous BLBP expression in the E12 forebrain and P6 cerebellum of transgenic mice. $(A)$ The construct used to assay promoter activity is similar to 9TM-0.8 (Feng and Heintz 1995) and contains Blbp genomic sequence from -766 to +53 , an intron containing a splice donor and acceptor (SD/SA), the E. coli $\beta$-galactosidase gene (LacZ), and a polyadenylation site $(\mathrm{pA})$. This construct drove efficient expression throughout the embryonic $(B, C)$ and post-natal $(D)$ CNS. Confirmation of the specificity of this construct was obtained by using the 766-bp fragment to drive eGFP; double labeling for BLBP and eGFP demonstrated that transgene expression is restricted to $\mathrm{BLBP}^{+}$cells in both the E12.5 forebrain $(E-G)$ and P6 cerebellum $(H)$. Arrows in $E$ and $F$ point to region shown at higher magnification in $G$.

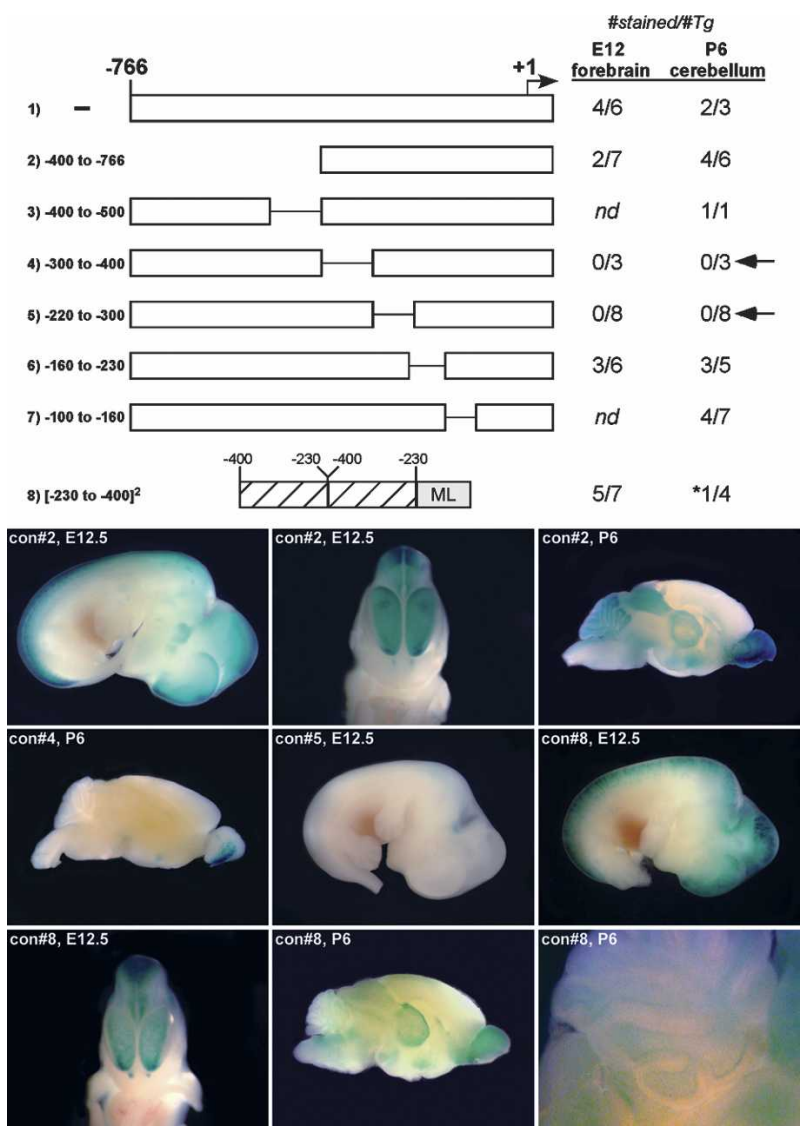

Figure 2. Elements between -230 and -400 are necessary and sufficient to drive Blbp transcription in the E12 forebrain and P6 cerebellum. A schematic of deletion (1-7) and transfer (8) constructs is shown. The ratios to the right indicate the number of independent transgenic founders that had detectable LacZ expression over the total number of founders obtained for a given construct. Of the constructs assayed, only deletions between -230 and -400 were observed to ablate expression (arrows). Representative whole-mount histochemical staining for $\beta$-galactosidase activity of founders are shown at the bottom; the construct used and assay time point is indicated in the upper left of each panel. $\left.{ }^{*}\right)$ The transfer construct expressed very strongly at E12 but rather weakly in the P6 cerebellum (histochemical staining is shown), suggesting that additional elements are required in the latter site to obtain normal transcriptional levels. (Tg) Transgenic founder; (nd) not determined.

and spatial patterns of transcription are driven from this 170-bp portion of the promoter, we dimerized and transferred it to the minimal adenovirus major late promoter (pML); this construct was called $[-230 \text { to }-400]^{2}$. The pML contains all of the basal elements required for transcription initiation in vitro but lacks sequences required for transcription in vivo (Miyamoto et al. 1984). Whereas transgenic founders containing $\mathrm{pML}$ alone showed no reporter expression (data not shown), those containing $[-230 \text { to }-400]^{2}$ displayed proper patterns of reporter activity throughout the E12 CNS as well as in P6 Bergmann glia (Fig. 2, construct 8). This demonstrates that the elements between -230 and -400 are both necessary and sufficient to direct $B l b p$ transcription in regions undergoing radial glial-directed neuronal migration. It is notable that similar to the construct containing only the first $400 \mathrm{bp}$ (construct 2), [-230 to -400$]^{2}$ drove strong and complete embryonic expression but only moderate restricted expression post-natally. This provides addi- 
tional evidence for developmental differences in the pathways that regulate $B 1 b p$ transcription and suggests that elements involved in the radial glial response to migrating neurons lie within -230 to -400 .

We next made a series of smaller deletions between -230 and -400 and assayed them in transgenic mice (Fig. $3)$. The only deletion that ablated reporter activity in both assay locations was the construct lacking the sequence between -230 and -265 (construct 12). Importantly, we noted that although this region is necessary for transcription both embryonically and post-natally, it is sufficient at neither time. This is evident from the fact that each of the other three deletions in the critical 170 bp caused loss of reporter expression at one of the two time points assayed (Fig. 3, constructs 9-11). These data suggest that a single signaling pathway may act throughout CNS development to induce transcription of $B l b p$ via elements lying between -230 and -265 , and that sequences flanking this domain respond to primarily to regionally and/or temporally restricted factors.

Analysis of the sequence between -230 and -265 revealed the presence of a 5'-GTTCCCAGGC-3' motif (Fig. 4A). This is a highly conserved binding site for the inducible transcription factor CBF1/RBP-Jк (Tun et al. 1994), the primary nuclear effector of signaling through the transmembrane Notch receptors. In the absence of adjacent cells expressing Notch-ligand, CBF1 associates with a corepressor complex to inhibit the transcription of Notch target genes. Upon interaction with cells displaying Notch ligands, the intracellular portion of Notch receptors is cleaved and translocates into the nucleus, where it physically associates with CBF1 to activate transcription of downstream genes (Lai 2002). Involve-

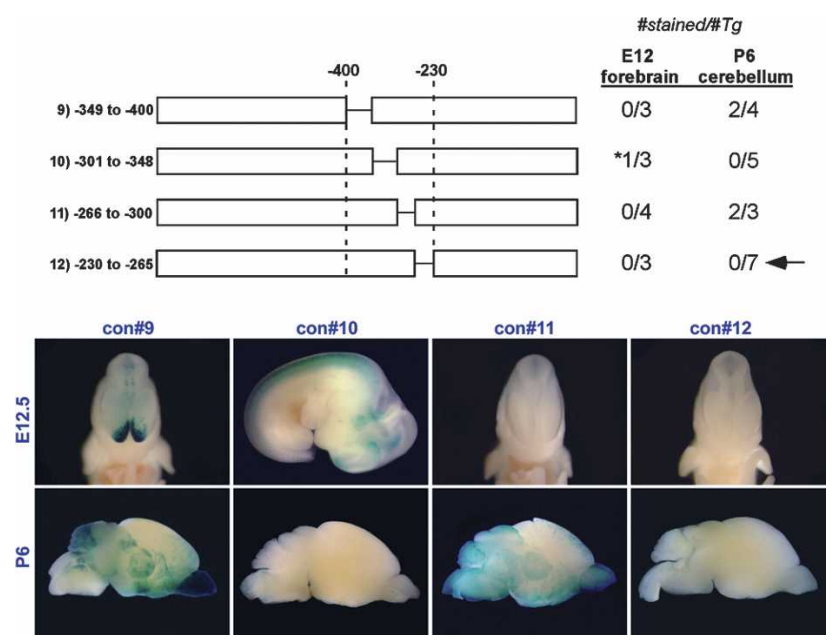

Figure 3. The sequence between -230 and -265 is essential for embryonic and post-natal transcription of Blbp. Finer deletions within the -230 to -400 interval defines the sequence between -230 and -265 (deletion 12, arrow) as critical for expression in both the E12 forebrain and P6 cerebellum. Note that the other three constructs drove expression at one of the two locations assayed, suggesting that elements in those intervals are mostly involved in regional and/or temporal control of Blbp transcription. Representative whole-mount histochemical staining for $\beta$-galactosidase activity of founders is shown at the bottom; the construct used and assay time point are indicated in the upper left of each panel. The strong expression in the developing olfactory bulbs seen at E12.5 for construct 9 was observed in two independent founders. $\left({ }^{*}\right)$ Construct 10 drove complete embryonic CNS expression but was noticeably weaker than the full-length 766-bp promoter.

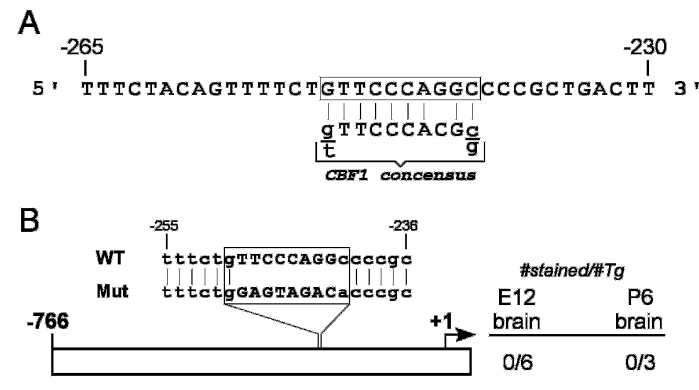

Figure 4. A consensus CBF1-binding site in the critical portion of the $B l b p$ promoter is required for transcriptional activity. $(A)$ The sequence of the critical interval between -230 and -265 contains a putative binding site for CBF1 (boxed); the homology between the Blbp CBF1-binding site and the published consensus sequence is indicated. $(B)$ Mutating the element in the context of the 766 promoter rendered the reporter construct completely inactive in both the E12 forebrain and P6 cerebellum; mice transgenic for the mutant CBF1-binding site were essentially identical in appearance (i.e., no detectable LacZ histochemical staining) to mice transgenic for construct 12 (Fig. 3).

ment of such a signaling pathway in Blbp transcription would be consistent with the observed inducibility of the Blbp gene in response to neuronal-glial contact (Feng and Heintz 1995), and with the cell-specific expression of Notch receptors in radial and Bergmann glia and Notch ligands in migrating neurons (Irvin et al. 2001; Solecki et al. 2001; Gaiano and Fishell 2002). The possible role of Notch signaling in Blbp transcription is also suggested by the findings that constitutive activation of Notch signaling up-regulates BLBP expression in radial glia in vivo (Gaiano et al. 2000) and post-natal cerebellar astroglia in vitro (Patten et al. 2003), and that dominant negative CBF1 represses transcription from the $B 1 b p$ promoter in cultured cerebellar astrocytes (Patten et al. 2003). To determine if the CBF1 cis-element is required for Blbp transcription in vivo, we mutated it in the context of the -766-bp promoter construct and assayed activity in transgenic mice. This resulted in a complete loss of promoter activity throughout the entire embryonic and post-natal brain (Fig. 4B), establishing that the CBF1 element is critical for Blbp transcription.

To further investigate the requirement of Notch/CBF1 signaling for Blbp transcription, we examined BLBP expression in mice lacking forebrain expression of the Notch 1 and Notch 3 receptors. These mice contain a conventional disruption in the Notch3 gene (Krebs et al. 2003) as well as selective deletion of a floxed Notch1 allele (Radtke et al. 1999) in the telencephalon using the Foxg1-Cre line (Hebert and McConnell 2000); thus, they lack Notch3 in all tissues, but Notch1 is absent only in those tissues that express Foxg1. Compared with wildtype embryos, conditional Notch1;Notch3 mutants (cNotch1;Notch3) had significantly reduced levels of BLBP in the E14.5 telencephalon (Fig. 5). This reduction was most prominent in the ganglionic eminences (GE) (Fig. 5A,I) but was also apparent in neocortex (the more obvious reduction of $B l b p$ expression in the GE is most likely due to the fact that ventral telencephalic radial glia contain much higher levels of BLBP protein than do neocortical radial glia in E14.5 wild-type mice) (Fig. 5E,M, arrowheads; Anthony et al. 2004). The decrease was not due to an absence of BLBP-expressing progenitor cells, as high magnification images of the ventricular 


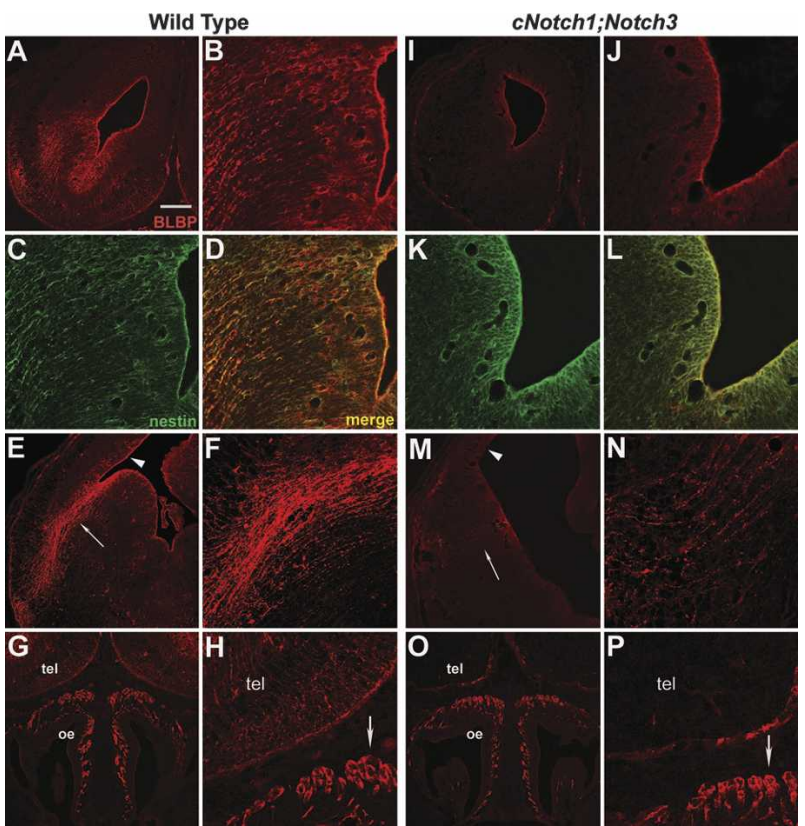

Figure 5. Radial glial expression of BLBP requires Notch1 and Notch3 signaling. Coronal sections of wild-type $(A-H)$ and cNotch1;Notch3 E14.5 mice $(I-P)$ immunostained for BLBP $(A, B, D-$ $J, L-P)$ and nestin $(C, D, K, L)$; sections in $A-D, G, H, I-L, O$, and $P$ are from the rostral forebrain, whereas those in $E, F, M$, and $N$ are at a more caudal level. BLBP expression is significantly reduced in the forebrains of cNotch1;Notch3 mice compared with wild type. $(J-L)$ This decrease is not due to an absence of BLBP-expressing progenitors, as nestin ${ }^{+}$cells containing low levels of BLBP can still be detected. In addition, cells with long radial processes are observed in mutant brains and stain weakly for BLBP (arrows in $E, M$ point to radial fibers shown at higher magnification in $F, N$, respectively), confirming that $B l b p$ transcription in radial glia requires Notch 1 and Notch3 signaling. $(G, H, O, P)$ Note that the reduction in BLBP expression is restricted to Foxg1-expressing regions (such as the telencephalon), whereas olfactory ensheathing glia contain equivalent levels of BLBP in both wild-type and mutant mice. Arrows in $H$ and $P$ point to olfactory ensheathing cells. (oe) Olfactory epithelium; (tel) telencephalon. Bars: $A, E, G, I, M, O, 200 \mu \mathrm{m} ; F, N, 80 \mu \mathrm{m}$; $B-D, H, P, 50 \mu \mathrm{m} ; J-L, 40 \mu \mathrm{m}$.

zone (VZ) revealed numerous nestin ${ }^{+}$cells containing low but detectable levels of BLBP in the mutants (Fig. 5J-L). It is noteworthy that although BLBP expression is strongly reduced in mutant brains, it is not entirely absent. Taken together with the complete loss of Blbp transcription observed in CBF1-binding site mutants (Fig. 4), these results raise the possibility that low levels of CBF1-mediated transcription can occur in the absence of Notch signaling in radial glia; support for such a possibility comes from the finding that the CBF1 ortholog $\mathrm{Su}(\mathrm{H})$ has a Notch-independent activation function during development of the external mechanosensory organs in Drosophila (Barolo et al. 2000). Alternatively, residual Blbp transcription could be due to compensation by Notch2, although this may be less likely given the low levels of Notch2 expression during early neurogenesis (H. Mason and G. Fishell, unpubl.) and opposite biological activities of Notch1 and Notch2 in tumorigenic neural progenitors (Fan et al. 2004).

Given that Notch signaling has been implicated in promoting a radial glial phenotype (Gaiano et al. 2000; Patten et al. 2003; Schmid et al. 2003), one explanation for the reduction of BLBP expression in cNotch1;Notch3 mutants is a lack of differentiated radial glial cells. However, cells with long radial processes were observed in mutant brains and contained $<38 \%$ of the level of BLBP observed in wild-type radial glia of the same region (for details on quantitation, see Materials and Methods; Fig. $5 \mathrm{E}, \mathrm{F}, \mathrm{M}, \mathrm{N})$. These results demonstrate that BLBP expression in radial glia requires Notch signaling. Finally, we note that the reductions of BLBP in cNotch1;Notch3 mutants are restricted to Foxg1-expressing regions (Fig. $5 \mathrm{G}, \mathrm{H}, \mathrm{O}, \mathrm{P})$, demonstrating that loss of BLBP expression is specific and restricted to regions with diminished Notch signaling.

The identification of Blbp as a Notch target indicates that the role of Notch signaling in neural progenitors varies as development proceeds, and that the spectrum of downstream target genes change. Previous work has shown that Blbp transcription in the neocortex does not begin until the onset of neurogenesis at E10.5, and that significant levels of BLBP protein are not detectable in this region until E12.5 (Anthony et al. 2004). In contrast, neocortical expression of Hes5 is detectable as early as E9.5 (Petersen et al. 2004), and high levels of both Notch1 and Hes5 are present by E10.5 (Yun et al. 2002). These data demonstrate that distinct developmental stages are accompanied by distinct patterns of Notch target gene expression. These shifts in Notch target gene expression appear to be mediated by additional regulatory factors that interact with the CBF1 coactivator complex; the existence of these additional factors is evident from our findings that the CBF1-binding site within the $B l b p$ promoter is necessary but not sufficient for transcription, and that multiple promoter elements mediate transcription of $B l b p$ at different times and places. POU domain transcription factors have previously been implicated in regulating $B 1 b p$ transcription in the embryonic forebrain (Josephson et al. 1998), and thus represent one possible class of $\mathrm{CBF} 1$-interacting proteins that function in radial glia. Interestingly, Notch and the POU domain protein Nubbin positively interact to promote gliogenesis in certain Drosophila cell lineages (Umesono et al. 2002). The fact that constitutively active Notch could promote glial fate in many but not all murine radial glia (Gaiano et al. 2000) may reflect a dependence of Notch signaling on other factors such as POU domain proteins. Additional candidates include factors downstream of Neuregulin and Reelin; these signaling molecules have previously been shown to induce radial glial expression of BLBP (Anton et al. 1997; Hartfuss et al. 2003).

Finally, we note that whereas radial glia serve as neuronal progenitors, Bergmann glia do not. This suggests that in addition to its well-documented role as a cell fate regulator, Notch signaling may also function to support neuronal migration. Previous studies have demonstrated that Notch signaling promotes a radial glial phenotype in the forebrain (Gaiano et al. 2000; Yoon et al. 2004), radializes cerebellar astrocytes (Patten et al. 2003), and induces expression of ErbB2, a receptor implicated in radial glial differentiation (Patten et al. 2003; Schmid et al. 2003). As antibody blocking experiments have implicated BLBP in regulating glial morphology (Feng et al. 1994; Anton et al. 1997; Miller et al. 2003), the available data suggest that Notch signaling may induce and/or maintain the radial glial scaffold, and that it does so in part through its induction of BLBP. Further insight into the mechanisms regulating radial glial function will 
likely be gained by the elucidation of BLBP function as well as the identification of additional radial glial Notch target genes.

\section{Materials and methods}

Constructs and generation of transgenic mice

All promoter constructs were made by PCRing from 9TM-0.8 (Feng and Heintz 1995); this plasmid contains -766 to +53 of the Blbp promoter in front of an intron, the LacZ gene, and a polyA cassette (Fig. 1). PCR was done using Expand High Fidelity PCR system (Roche), and products were cloned back into the pNASS $\beta$ vector (Clontech) between the EcoRI and Xhol sites. The transfer construct was made by placing two 170-bp PCR fragments (from -230 to -400 of the Blbp promoter) in front of the minimal adeno major late promoter (ML), which extends from -40 to +66 of the adenovirus major late promoter (Miyamoto et al. 1984); in the absence of additional promoter/enhancer sequences, ML does not express at all in transgenic mice (data not shown). All deletions and mutations were confirmed by DNA sequencing. For generation of transgenic mice, conventional transgene DNA was digested to remove vector sequences, isolated from low melting gels using Qiaquick columns (Qiagen), and further purified using Elutip-d columns (Schleicher and Schuell). Transgenic B6CBA mice were generated using standard protocols (Hogan et al. 1994). All mice were typed by PCR of DNA from yolk sacs (embryos) or tails (post-natal).

\section{Deletion of Notch1 and Notch3}

Floxed Notch1 mice were a gift of Freddy Radtke (Ludwig Institute for Cancer Research, Lausanne Branch, University of Lausanne, Epalinges, Sweden) and were genotyped as previously described (Radtke et al. 1999). Mutant mouse embryos were obtained by crossing homozygous floxed Notch1 mice with mice heterozygous for floxed Notch1 and Foxg $1^{\text {Cre/+ }}$. The generation of Foxg $1^{\text {Cre/+ }}$ knockin mice was previously published (Hebert and McConnell 2000) and were maintained as heterozygotes on a Swiss Webster background. The generation of Notch3 null mutant mice was previously described (Krebs et al. 2003). Conditional Notch1;Notch3 double mutant mice were obtained by breeding double homozygous floxed Notch1;Notch3 null mutant mice with mice heterozygous for floxed Notch1 and Foxg1 ${ }^{\mathrm{Cre} /+}$ on a Notch3 null mutant background.

\section{X-Gal histochemistry}

Tissues were immersion fixed in $4 \%$ paraformaldehyde for $15 \mathrm{~min}$ at room temperature and incubated overnight at $37^{\circ} \mathrm{C}$ in X-Gal solution: 1 $\mathrm{mg} / \mathrm{mL} \mathrm{X-gal} \mathrm{(Sigma),} 20 \mathrm{mM} \mathrm{K}{ }_{3} \mathrm{Fe}(\mathrm{CN})_{6}, 20 \mathrm{mM} \mathrm{K}_{4} \mathrm{Fe}(\mathrm{CN})_{6}, 2 \mathrm{mM}$ $\mathrm{MgCl}_{2}$, and $0.02 \% \mathrm{NP}-40$ in PBS.

\section{Immunofluorescent staining and quantification}

Vibratome or cryostat sections were stained with the following antibodies: rabbit $\alpha$-BLBP $(1: 2000)$ (Feng et al. 1994), mouse $\alpha$-nestin/rat-401 (1:4, Developmental Studies Hybridoma Bank), rabbit $\alpha$ - $\beta$-galactosidase (1:500, ICN), and goat $\alpha$-GFP (1:500, US Biological). Secondary antibodies used at 1:500 (Cy2 and Cy3) were from Jackson Immunoresearch. Confocal imaging of wild-type and cNotch1;Notch3 tissues was done on an LSM 510 Axioplan (Zeiss) using identical settings; under the imaging parameters used, pixel saturation was not observed. To quantify the reduction of BLBP expression in cNotch1;Notch3 mice, MetaMorph Imaging System software (Universal Imaging Corporation) was used to determine the intensity of immunfluorescent staining present in individual radial glial processes in the GE. Normalization for possible variability in tissue processing (which could affect staining intensities) was done by comparing BLBP immunostaining in radial glia to that in olfactory ensheathing glial cells (OEG); since Foxg1 is not expressed in these cells, Notch1 expression is not affected. Therefore, OEG should contain essentially equivalent levels of BLBP in both genotypes and serve as an internal control. Measurements obtained (in average intensity $/ \mu^{2} \pm$ standard deviation) were as follows: wild-type OEG, $89.1 \pm 3.3(n=22)$; wild-type GE, $84.2 \pm 3.9(n=21)$; cNotch1;Notch3 OEG, $88.9 \pm 3.4(n=20)$; and cNotch1;Notch3 GE, $31.6 \pm 9.1(n=18)$.

\section{Acknowledgments}

We thank Dr. Mary-Beth Hatten for very helpful discussions during the course of this study, as well as for the use of laboratory equipment and for providing reagents. N.H. is an HHMI Investigator; T.A. was supported by a National Institutes of Health $(\mathrm{NIH})$ genetics predoctoral training grant and HHMI; and G.F. is supported by the NIH, a March of Dimes basic research grant, and a Children's Brain Tumor Foundation grant.

\section{References}

Angevine, J.B. and Sidman, R.L. 1961. Autoradiographic study of cell migration during histogenesis of cerebral cortex in the mouse. Nature 192: 766-768.

Anthony, T.E., Klein, C., Fishell, G., and Heintz, N. 2004. Radial glia serve as neuronal progenitors in all regions of the central nervous system. Neuron 41: 881-890.

Anton, E.S., Marchionni, M.A., Lee, K.F., and Rakic, P. 1997. Role of GGF/neuregulin signaling in interactions between migrating neurons and radial glia in the developing cerebral cortex. Development 124: 3501-3510.

Barolo, S., Walker, R.G., Polyanovsky, A.D., Freschi, G., Keil, T., and Posakony, J.W. 2000. A notch-independent activity of suppressor of hairless is required for normal mechanoreceptor physiology. Cell 103: 957-969.

Fan, X., Mikolaenko, I., Elhassan, I., Ni, X., Wang, Y., Ball, D., Brat, D.J., Perry, A., and Eberhart, C.G. 2004. Notch1 and notch2 have opposite effects on embryonal brain tumor growth. Cancer Res. 64: 77877793.

Feng, L. and Heintz, N. 1995. Differentiating neurons activate transcription of the brain lipid-binding protein gene in radial glia through a novel regulatory element. Development 121: 1719-1730.

Feng, L., Hatten, M.E., and Heintz, N. 1994. Brain lipid-binding protein (BLBP): A novel signaling system in the developing mammalian CNS. Neuron 12: 895-908.

Gaiano, N. and Fishell, G. 2002. The role of notch in promoting glial and neural stem cell fates. Annu. Rev. Neurosci. 25: 471-490.

Gaiano, N., Nye, J.S., and Fishell, G. 2000. Radial glial identity is promoted by Notch1 signaling in the murine forebrain. Neuron 26: 395 404.

Hartfuss, E., Forster, E., Bock, H.H., Hack, M.A., Leprince, P., Luque, J.M., Herz, J., Frotscher, M., and Gotz, M. 2003. Reelin signaling directly affects radial glia morphology and biochemical maturation. Development 130: 4597-4609.

Hatten, M.E. 1985. Neuronal regulation of astroglial morphology and proliferation in vitro. J. Cell. Biol. 100: 384-396.

. 1999. Central nervous system neuronal migration. Annu. Rev. Neurosci. 22: 511-539.

Haunerland, N.H. and Spener, F. 2004. Fatty acid-binding proteins: Insights from genetic manipulations. Prog. Lipid Res. 43: 328-349.

Hebert, J.M. and McConnell, S.K. 2000. Targeting of cre to the Foxg1 (BF-1) locus mediates loxP recombination in the telencephalon and other developing head structures. Dev. Biol. 222: 296-306.

Hogan, B., Beddington, R., Costantini, F., and Lacy, E. 1994. Manipulating the mouse embryo: A laboratory manual. Cold Spring Harbor Laboratory Press, Cold Spring Harbor, NY.

Irvin, D.K., Zurcher, S.D., Nguyen, T., Weinmaster, G., and Kornblum, H.I. 2001. Expression patterns of Notch1, Notch2, and Notch3 suggest multiple functional roles for the Notch-DSL signaling system during brain development. J. Comp. Neurol. 436: 167-181.

Josephson, R., Muller, T., Pickel, J., Okabe, S., Reynolds, K., Turner, P.A., Zimmer, A., and McKay, R.D. 1998. POU transcription factors control expression of CNS stem cell-specific genes. Development 125: 3087-3100.

Krebs, L.T., Xue, Y., Norton, C.R., Sundberg, J.P., Beatus, P., Lendahl, U., Joutel, A., and Gridley, T. 2003. Characterization of Notch3-deficient mice: Normal embryonic development and absence of genetic interactions with a Notch1 mutation. Genesis 37: 139-143.

Kurtz, A., Zimmer, A., Schnutgen, F., Bruning, G., Spener, F., and Muller, T. 1994. The expression pattern of a novel gene encoding brain-fatty acid binding protein correlates with neuronal and glial cell development. Development 120: 2637-2649.

Lai, E.C. 2002. Keeping a good pathway down: Transcriptional repression of Notch pathway target genes by CSL proteins. EMBO Rep. 3: 840 845 .

Malatesta, P., Hartfuss, E., and Gotz, M. 2000. Isolation of radial glial cells by fluorescent-activated cell sorting reveals a neuronal lineage. 
Development 127: 5253-5263.

Miller, S.J., Li, H., Rizvi, T.A., Huang, Y., Johansson, G., Bowersock, J., Sidani, A., Vitullo, J., Vogel, K., Parysek, L.M., et al. 2003. Brain lipid binding protein in axon-Schwann cell interactions and peripheral nerve tumorigenesis. Mol. Cell. Biol. 23: 2213-2224.

Miyamoto, N.G., Moncollin, V., Wintzerith, M., Hen, R., Egly, J.M., and Chambon, P. 1984. Stimulation of in vitro transcription by the upstream element of the adenovirus-2 major late promoter involves a specific factor. Nucleic Acids Res. 12: 8779-8799.

Miyata, T., Kawaguchi, A., Okano, H., and Ogawa, M. 2001. Asymmetric inheritance of radial glial fibers by cortical neurons. Neuron 31: $727-$ 741.

Noctor, S.C., Flint, A.C., Weissman, T.A., Dammerman, R.S., and Kriegstein, A.R. 2001. Neurons derived from radial glial cells establish radial units in neocortex. Nature 409: 714-720.

Patten, B.A., Peyrin, J.M., Weinmaster, G., and Corfas, G. 2003. Sequential signaling through Notch1 and erbB receptors mediates radial glia differentiation. J. Neurosci. 23: 6132-6140.

Petersen, P.H., Zou, K., Krauss, S., and Zhong, W. 2004. Continuing role for mouse Numb and Numbl in maintaining progenitor cells during cortical neurogenesis. Nat. Neurosci. 7: 803-811.

Radtke, F., Wilson, A., Stark, G., Bauer, M., van Meerwijk, J., MacDonald, H.R., and Aguet, M. 1999. Deficient T cell fate specification in mice with an induced inactivation of Notch1. Immunity 10:547-558.

Rio, C., Rieff, H.I., Qi, P., Khurana, T.S., and Corfas, G. 1997. Neuregulin and erbB receptors play a critical role in neuronal migration. Neuron 19: $39-50$.

Schmid, R.S., McGrath, B., Berechid, B.E., Boyles, B., Marchionni, M., Sestan, N., and Anton, E.S. 2003. Neuregulin 1-erbB2 signaling is required for the establishment of radial glia and their transformation into astrocytes in cerebral cortex. Proc. Nat1. Acad. Sci. 100: 42514256.

Sidman, R.L. and Rakic, P. 1973. Neuronal migration, with special reference to developing human brain: A review. Brain Res. 62: 1-35.

Solecki, D.J., Liu, X.L., Tomoda, T., Fang, Y., and Hatten, M.E. 2001. Activated Notch2 signaling inhibits differentiation of cerebellar granule neuron precursors by maintaining proliferation. Neuron 31: 557568.

Tamamaki, N., Nakamura, K., Okamoto, K., and Kaneko, T. 2001. Radial glia is a progenitor of neocortical neurons in the developing cerebral cortex. Neurosci. Res. 41: 51-60.

Tun, T., Hamaguchi, Y., Matsunami, N., Furukawa, T., Honjo, T., and Kawaichi, M. 1994. Recognition sequence of a highly conserved DNA binding protein RBP-J к. Nucleic Acids Res. 22: 965-971.

Umesono, Y., Hiromi, Y., and Hotta, Y. 2002. Context-dependent utilization of Notch activity in Drosophila glial determination. Development 129: 2391-2399.

Yoon, K., Nery, S., Rutlin, M.L., Radtke, F., Fishell, G., and Gaiano, N 2004. Fibroblast growth factor receptor signaling promotes radial glial identity and interacts with Notch1 signaling in telencephalic progenitors. J. Neurosci. 24: 9497-9506.

Yun, K., Fischman, S., Johnson, J., De Angelis, M.H., Weinmaster, G., and Rubenstein, J.L. 2002. Modulation of the notch signaling by Mash1 and Dlx1/2 regulates sequential specification and differentiation of progenitor cell types in the subcortical telencephalon. Development 129: 5029-5040.

Zheng, C., Heintz, N., and Hatten, M.E. 1996. CNS gene encoding astrotactin, which supports neuronal migration along glial fibers. Science 272: 417-419. 


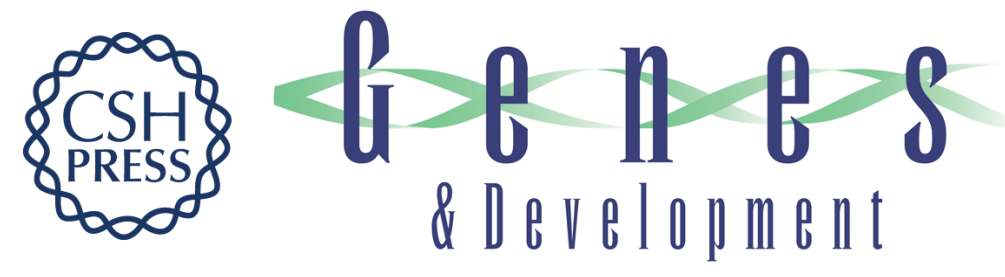

\section{Brain lipid-binding protein is a direct target of Notch signaling in radial glial cells}

Todd E. Anthony, Heather A. Mason, Thomas Gridley, et al.

Genes Dev. 2005, 19:

Access the most recent version at doi:10.1101/gad.1302105

References This article cites 36 articles, 15 of which can be accessed free at: http://genesdev.cshlp.org/content/19/9/1028.full.html\#ref-list-1

License

Email Alerting

Receive free email alerts when new articles cite this article - sign up in the box at the top Service right corner of the article or click here.

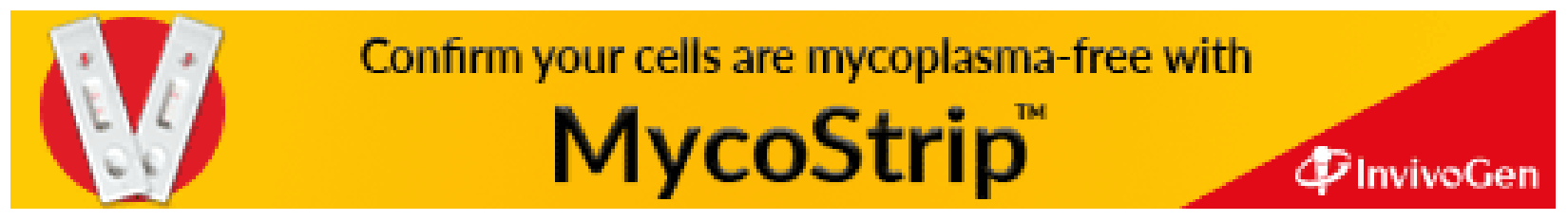

